\title{
Cholesterol Granuloma of Omentum Comformed by Ultrasound-Guided Biopsy: A Case Report
}

\author{
Zhiguang Chen, $\mathrm{MD}^{a}$, Xuemei Wang, MD ${ }^{a}$, Liang Sang, MD ${ }^{a \text {,* }}$ \\ ${ }^{a}$ Department of Ultrasound, The First Hospital of China Medical University, Shenyang, Liaoning Province, China \\ Received October 04, 2019; revision received January 14, 2020; accepted February 02, 2020
}

\begin{abstract}
Cholesterol granuloma (CG) is a histological lesion caused by an inflammatory reaction of the cholesterol crystal rather than an independent disease. We report the case of an 87-year-old woman who came to our hospital for slight abdominal pain lasting a week and who had a history of a pelvic mass found forty years ago. Ultrasound examination revealed abnormal omentum, which was suspected to be a source of metastasis from the pelvic mass. However, ultrasound-guided biopsy confirmed the abnormal omentum was CG. By describing this case, we aim to share the value of ultrasound-guided omentum biopsy in clinical diagnosis and treatment.
\end{abstract}

Key words: Cholesterol granuloma; Ultrasound-guided biopsy; Omentum

Advanced Ultrasound in Diagnosis and Therapy 2020;03:248-250

DOI: 10.37015/AUDT.2020.190026

\section{Case Report}

In May 2018, an 87-year-old woman came to our hospital with abdominal pain, lasting for about a week, accompanied by nausea and vomiting. Forty years ago, the patient had a small pelvic mass incidentally found through ultrasonography, but the characterization of the mass and the process of the treatment were not clear. A week ago, digestive symptoms started to appear with more serious abdominal pain. Routine physical examination after admission showed that the lower abdomen was slightly convex, mild tenderness was present without rebound pain, and Murphy's sign was negative. Gynecological examination revealed the vulva was normal, a small amount of vaginal bleeding, and a mass of the size of the head of a newborn in the pelvic cavity with poorly mobility

Ultrasound (US) examination revealed a mixed-echo block behind the uterus (approximately $18.20 \times 8.94 \times$ $15.06 \mathrm{~cm}$ in size), and abnormally thickened omentum was present in the right upper abdomen with a highlow mixed echo nodule (Fig. 1A). The structure of the thickened omentum was compact. Color Doppler flow imaging (CDFI) showed poor blood flow with points and short strip-like blood flow signals (Fig. 1B). In addition, ascites could be seen in the abdominal cavity. Realtime tissue elastography (RTE) was classified according the following standard: a score of 1 indicated that the sampling frame was uniformly green; 2 indicated that the sampling frame was composed of blue and green, mainly green; 3 indicated that the sampling frame was composed of blue and green, mainly blue; and 4 indicated that the sampling frame was uniformly blue. The nodules had an elasticity score of 3 (Fig. 2A). The ultrasound system can track the displacement to calculate the shear wave propagation speed and the quantitative tissue stiffness (i.e., Young's modulus, $\mathrm{kPa}$ ). Shear wave elastography (SWE) is a novel real-time imaging technique that represents a substantial advance in ultrasound elastography. In this case, the mean Young's modulus value was $50.9 \mathrm{kPa}$ (Fig. 2B). Computerized tomography $(\mathrm{CT})$ revealed that the original intestinal mass could be a malignancy and that the newly identified nodule of the omentum might be a metastasis (Fig. 3). Ultrasound-guided biopsy of the nodule in the omentum was performed.

According to the pathological outcome, under

* Corresponding author: Department of Ultrasound, The First Hospital of China Medical University, Shenyang 110001, Liaoning Province, China.

e-mail:13889167622@163.com 
the optical microscope, histocyte and epithelial cells aggregated together with inflammatory cells' infiltration, and cholesterol crystals appeared in the interstitial spaces (Fig. 4A). Immunohistochemistry showing CK (-), Vimentin (+), CD68 (+), P63 (-), CK5/6 (-), S-100 (-), and $\mathrm{Ki}-67$ (about $20 \%+$ ) was consistent with cholesterol granuloma (CG) of omentum (Fig. 4B). Because the patient could not tolerate surgical trauma, her family declined further examination and treatment.
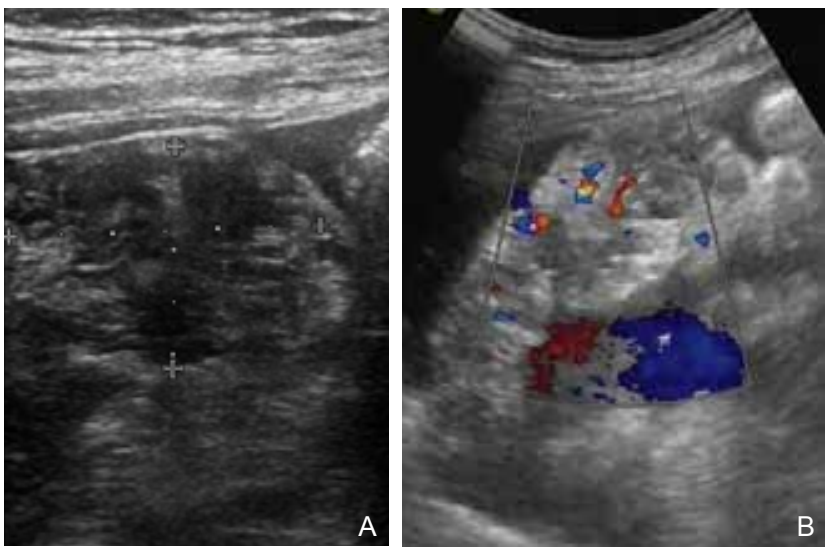

Figure 1 US examination. (A) High frequency linear array probe imaging showed thickened omentum with mixed echogenic appearance; (B) Low frequency convex array probe imaging of color Doppler flow showed the thickened omentum with minimal blood flow signals.
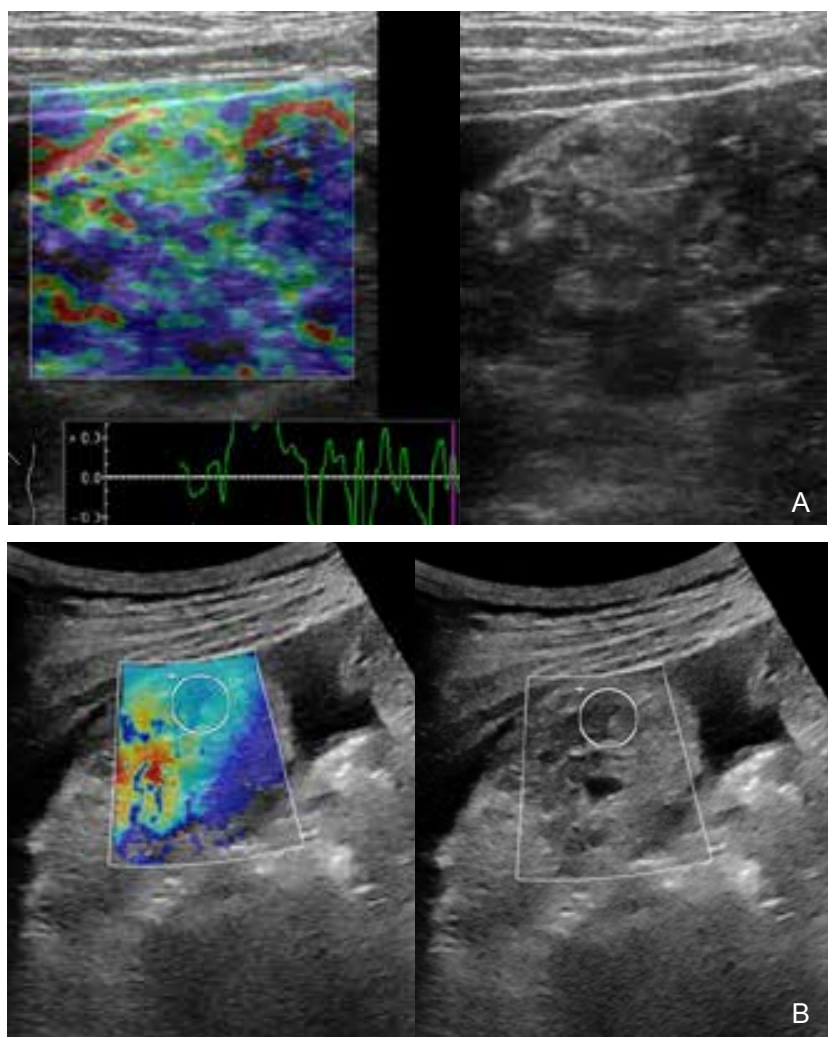

Figure 2 US elastography. (A) Real-time tissue elastography showed that the nodules of the omentum had elasticity scoring of 3; (B) Share wave elastography measurement of the omentum mass was $50.9 \mathrm{kPa}$.

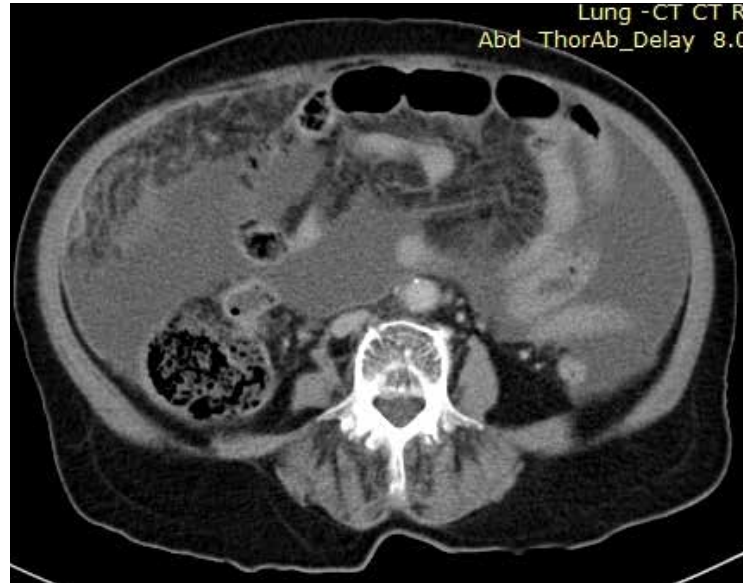

Figure 3 Computerized tomography showed that the omentum in the right upper abdomen was thickened neoplasm.
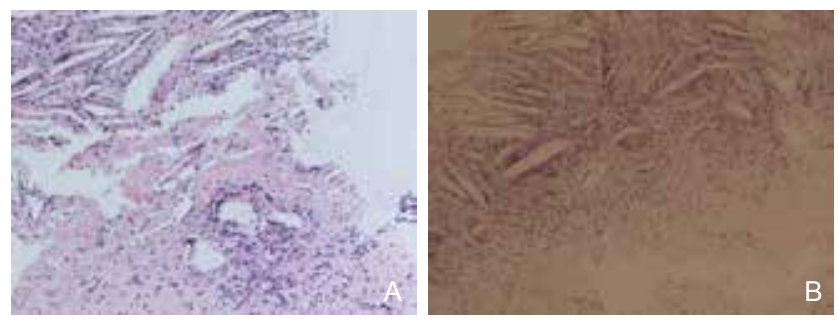

Figure 4 Pathological findings. (A) Optical microscope showed histocytes, epithelial cells aggregation, inflammatory cells infiltrate, and cholesterol crystals appeared in the interstitial tissue; (B) Immunohistochemistry showed CK (-), Vimentin (+), CD68(+), P63 (-) CK5/6 (-), S-100 (-), and Ki-67 (about 20\%+).

\section{Discussion}

$\mathrm{CG}$ is not an independent disease but a histological lesion that is caused by an inflammatory reaction of the cholesterol crystals. It could occur in any part of the temporal bone, but most commonly occurs in the middle ear; it is rare to find it elsewhere, especially in the omentum [1]. CG is composed of cholesterol crystal and giant cells, with microscopic examination showing a large number of cholesterol crystals surrounded by heterogeneous multinucleated giant cells, histiocytes, and a large number of lymphocytes [2]. Submucosal hemorrhage and serum exudates into the sealed structure of the body and the red blood cells (RBCs) decompose and produce cholesterol crystals that induce foreign body reactions, including multinucleated giant cells and macrophage aggregation, hyperplasia of blood vessels and fibrous tissue, and formation of the cholesterol granuloma [3].

The patient had a chronic disease history, and the pelvic mass appears to have caused the immune system to remain in a highly sensitive state and cause a local inflammatory reaction. Simultaneously, the existence of chronic inflammation in the omentum led to the 
exudation of the lymphocytes and RBC. With time, more and more decomposed RBCs accumulated in the omentum. Therefore, the affected part of the omentum was hard and stiff.

According to Zhang et al. [4], the elasticity scores of malignantly thickened omentum are higher than for benign thickened omentum, with more than $90 \%$ of the malignantly thickened omentum having elasticity scores of 3 or 4 . Because this patient had an elasticity score of 3, we therefore thought the thickened omentum was malignant. Furthermore, since ultrasound elastography (UE) was introduced by Ophir et al. [5], it has been widely used in the diagnosis and differential diagnosis of diseases such as thyroid, breast, prostate and liver [6]. According to Ophir et al study, the Young's modulus value of liver increased as the liver hardness increased to evaluate the hardness of the abnormal omentum [7]. We considered the Young's modulus value of $50.9 \mathrm{kPa}$ for this omentum as hard tissue. Based on these results and integrating the examination of CT and UE, the authors believed the lesions of the omentum mostly transferred from the pelvic lesions. However, ultrasound-guided biopsy revealed it to be CG instead.

\section{Conclusion}

Notably, although the patient's ultrasound of the omentum suggested a possible malignancy, it is difficult to discern whether a mass is benign or malignant through imaging alone. Thus, ultrasound-guided biopsy of the omentum is an indispensable method for assisting diagnosis of an indeterminate mass.

\section{Conflict of Interest}

The authors report no conflict of interest in this work.

\section{References}

[1] Shrirao N, Mukherjee B, Krishnakumar S, Biswas J. Cholesterol granuloma: a case series \& review of literature. Graefes Arch Clin Exp Ophthalmol 2016;254:185-188.

[2] Sadé J, Halevy A, Klajman A, Mualem T. Cholesterol granuloma. Acta Otolaryngol 1980;89:233-239.

[3] Januszek G, Niemczyk K, Morawski K, Bartoszewicz R, OrkanLecka E. Cholesterol granuloma. Otolaryngol Pol 2007;61:637-42.

[4] Zhang Y, Wang X, Tao C, Que Y, Zhao W, Chen B. Ultrasound elastography for differentiating benign from malignant thickened greater omentum. Eur Radiol 2016;26:2337-2343.

[5] Ophir J, Céspedes I, Ponnekanti H, Yazdi Y, Li X. Elastography: a quantitative method for imaging the elasticity of biological tissues. Ultrason Imaging 1991;13:111-134.

[6] Sigrist RMS, Liau J, Kaffas AE, Chammas MC, Willmann JK. Ultrasound Elastography: Review of Techniques and Clinical Applications. Theranostics 2017;7:1303-1329.

[7] Herrmann E, de Lédinghen V, Cassinotto C, Chu WC, Leung VY, Ferraioli G, et al. Assessment of biopsy-proven liver fibrosis by twodimensional shear wave elastography: An individual patient databased meta-analysis. Hepatology 2018;67:260-272. 